\title{
Lothar Gall
}

\section{Adel, Verein und städtisches Bürgertum}

Für die Entwicklung der europäischen Staaten und ihrer Gesellschaften ist es offenkundig von zentraler Bedeutung gewesen, wie sich im Zuge des stürmischen gesellschaftlichen Wandels seit dem 18. Jahrhundert das Verhältnis zwischen dem Adel und den führenden Kräften des aufstrebenden Bürgertums entwickelte, ob in Richtung auf einen Elitenkonflikt oder auf einen Elitenkompromiß. Dem zunächst klassischen Land des Elitenkonfliktes, dem Frankreich der großen Revolution, stehen England, Italien und, mit etwas anderen Akzenten, Deutschland als Gebiete gegenüber, in denen nach weit verbreiteter Meinung die Tendenzen zum Elitenkompromiß überwogen. Im Falle Deutschlands hat man dabei allerdings vor allem Preußen vor Augen, und hier insbesondere die Zeit nach 1850. Wie sich die Dinge in den Jahrzehnten davor, zumal im außerpreußischen Deutschland, darstellten, ist hingegen bisher kaum untersucht worden ${ }^{1}$.

Eines ist freilich ganz deutlich und markiert die Ausgangslage: Der Gegensatz zum Adel war wie fast überall in Europa so auch in Deutschland insofern einer der entscheidenden Faktoren bei der Konstituierung des Bürgertums als einer überlokalen und überregionalen Einheit, als sich aus der Frontstellung zum Adel, die sich nicht

1 Allgemein zum Adel in der Phase des Übergangs von der ständischen zur bürgerlichen Gesellschaft in Mitteleuropa: Karl-Georg Faber, Mitteleuropäischer Adel im Wandel der Neuzeit, in: GG 7 (1981) 276-296; Heinz Reif, Der Adel in der modernen Sozialgeschichte, in: Wrolfgang Schieder/Volker Sellin (Hrsg.), Sozialgeschichte in Deutschland. Bd. IV: Soziale Gruppen in der Geschichte (Göttingen 1986) 34-60; Armgard von Reden-Dobna/Ralph Melville (Hrsg.), Der Adel an der Schwelle des bürgerlichen Zeitalters 1780-1860 (Stuttgart 1988); Elisabeth Febrenbach, Der Adel in Frankreich und Deutschland im Zeitalter der Französischen Revolution, in: Helmut Berding/Etienne Frangois/Hans-Peter Ullmann (Hrsg.), Deutschland und Frankreich im Zeitalter der Französischen Revolution (Frankfurt 1989) 177-215; Hans-Ulrich Webler (Hrsg.), Europäischer Adel 1750-1950 (Göttingen 1990); Karl Möckl (Hrsg.), Hof und Hofgesellschaft in den deutschen Staaten im 19. und beginnenden 20. Jahrhundert (Boppard a. Rhein 1990) sowie als wegweisende Fallstudien Heinz Reif, Westfälischer Adel 1770-1860. Vom Herrschaftsstand zur regionalen Elite (Göttingen 1979) u. Heinz Gollwitzer, Die Standesherren. Die politische und gesellschaftliche Stellung der Mediatisierten 1815-1918 (Göttingen ${ }^{2} 1964$ ). S. a. Christoph Arneke, Untersuchungen zur Demographie des niederen Adels in Deutschland im 19. Jahrhundert (Düsseldorf 1984); Gregory W. Pedlow, The Survival of the Hessian Nobility, 1770-1870 (Princeton 1988); Robert M. Berdabl, The Politics of the Prussian Nobility - The Development of a Conservative Ideology 1770-1848 (Princeton 1988) sowie die verschiedenen Arbeiten von Walter Demel z. bayerischen Adel, zusammenfassend zuletzt in Hans-Ulricb Webler (Hrsg.), Europäischer Adel (wie eben zit.), $126 \mathrm{ff}$. 
selten zu einem dezidierten Antiaristokratismus steigerte, das Bewußtsein der inneren Einheit der eigenen sozialen Gruppe nährte. Selbstverständnis und Selbstbewußtsein, politische und soziale Erwartungen und Zielvorstellungen, Auftreten und Lebensstil all das wurde sehr wesentlich geprägt in der Auseinandersetzung mit dem Adel und der oft heftigen Konfrontation mit dessen Ansprüchen, auch mit dessen gerade in Mitteleuropa, im Alten Reich, besonders ausgeprägtem Beharren auf Exklusivität und Abgrenzung. Aus der Geistes- und Literaturgeschichte insbesondere des 18. Jahrhunderts ist das sattsam bekannt: Man denke an Lessing und vor allem an Schiller, der mit seinen Dramen wie in seiner Person geradezu ein Modell für bürgerliches Aufbegehren gegen die Anmaßungen der Aristokratie bot.

Was in vielfältigen Abwandlungen in dieser Hinsicht auf dem Theater und im Roman präsentiert wurde, entsprach in der Tendenz den lebensweltlichen Erfahrungen, die viele Vertreter des Bürgertums, insbesondere in den Bildungsberufen, machten, also als Hauslehrer, als Geistliche, an den Universitäten, im Fürstendienst, im Hinblick auf die Rangordnungen der Hofgesellschaften. Im zweiten Teil des bekannten Goetheschen „Wo kam die schönste Bildung her/ Und wenn sie nicht vom Bürger wär" wird der oft quälend empfundene Widerspruch zwischen Anspruch und Lebenswirklichkeit schlaglichtartig beleuchtet, wenn es dort heißt: „Wenn aber sich Ritter und Bauern verbinden/ Da werden sie freilich die Bürger schinden. ${ }^{“ 2}$

Die wachsenden, in der Literatur vielfältig akzentuierten Spannungen zwischen dem Adel und Vertretern des sogenannten Bildungsbürgertums stellten freilich insgesamt gesehen doch mehr Reibungen an der jeweiligen Peripherie von Adels- und Bürgerwelt dar. Bis tief ins 19. Jahrhundert hinein bildeten diese beiden Welten sehr deutlich voneinander geschiedene „sozial-moralische Milieus“, um den von R. M. Lepsius für die Parteienklientel des 19. und der ersten Jahrzehnte des 20. Jahrhunderts geprägten Begriff aufzugreifen. Ihre Berührungsflächen beschränkten sich auf wenige Bereiche. In diesen Bereichen, in den „Kontaktzonen“ (E. Fehrenbach) zwischen den beiden Milieus entschied sich allerdings, nicht zuletzt durch die Wirkungen, die davon auf beide Lager und deren Grundeinstellungen ausgingen, die weitere Entwicklung des Verhältnisses zwischen den beiden Führungsschichten, der alten aristokratischen und der neu aufstrebenden bürgerlichen Elite. Insofern kommt der Frage, wie sich hier die Dinge seit dem Ausgang des 18. Jahrhunderts gestaltet haben, grundsätzliche Bedeutung zu. So haben es im übrigen auch schon viele Zeitgenossen gesehen.

Hier: das heißt wo Adel und Bürgertum konkret zusammenlebten, wo sich ihre Lebenswelten unmittelbar berührten und ihre jeweiligen Daseinsvorstellungen und Lebensansprüche direkt in Kontakt und gegebenenfalls in Konflikt miteinander gerieten, also im Bereich der Stadt, genauer gesagt bestimmter Städte, in denen der Adel zahlenmäßig stärker vertreten war. Das waren in erster Linie die Residenzstädte bzw. auch ehemalige Residenzstädte wie zum Beispiel Mannheim sowie Garnisonsstädte und regionale Verwaltungszentren. In ihnen bildeten Angehörige des Adels als Beamte, als Offiziere, als Mitglieder der Hofgesellschaft einen erheblichen Teil der gesellschaftlichen Führungsschicht der jeweiligen Stadt mit vielfältigen Berührungen

\section{Zahme Xenien, IX.}


mit ihrem bürgerlichen Pendant, mit den führenden Gruppen des städtischen Bürgertums. Die Kontakte zwischen den beiden „Fraktionen“ konzentrierten sich vor allem $\mathrm{da}$, wo sich, wie wir inzwischen aus zahlreichen Untersuchungen wissen, überall in den Städten die Führungsschicht zusammenfand und als solche informell konstituierte: in den allgemeinen „bürgerlichen“ Vereinen, den Casino-, Museums-, Harmoniegesellschaften oder "Civilclubs“, die seit Ausgang des 18. Jahrhunderts in praktisch jeder größeren mitteleuropäischen Stadt ins Leben traten ${ }^{3}$. Sie bilden das sich unmittelbar anbietende Untersuchungsfeld für die Beantwortung der Frage, ob sich Adel und Bürgertum, genauer gesagt die alte aristokratische und die neue bürgerliche Elite, aufeinander zubewegten, zu Symbiose und Elitenkompromiß tendierten, oder ob sie in Distanz zueinander verharrten, auf Abgrenzung bedacht blieben und gegebenenfalls eher zum Konflikt neigten.

Vor Eintritt in die Untersuchung bedarf es dabei noch der Klärung einer Vorfrage: Wo ist bei der Bestimmung beider Fraktionen die alte städtische Führungsschicht, das sogenannte Patriziat, einzuordnen: beim Adel, dem sich seine Angehörigen im Ancien Régime vielfach zugehörig, freilich oft auch überlegen fühlten, oder beim Bürgertum, dessen führende Vertreter sie - man denke an Hamburg, an Frankfurt, an Köln, Augsburg oder Nürnberg - ja ebenfalls zu sein beanspruchten und verfassungsrechtlich auch oft waren? Mit Ausnahme der Hansestädte, wo sich das Patriziat, unter Aufnahme neuer Elemente, praktisch das ganze 19. Jahrhundert hindurch als Führungsschicht behauptete - und dabei immer sehr stark seinen bürgerlichen Charakter betonte $^{4}$ - läßt sich die Frage seit dem großen Umbruch der Wende vom 18. zum 19. Jahrhundert mit seinen tiefgreifenden Wirkungen auch auf die Verfassungen der Städte jeweils nur noch individuell, von den Optionen seiner einzelnen, als Gruppe durchgängig politisch entmachteten Mitglieder entscheiden. Einige, so macht etwa das

${ }^{3}$ Zur Bedeutung der Vereine grundlegend Thomas Nipperdey, Verein als soziale Struktur in Deutschland im späten 18. und frühen 19. Jahrhundert, in: ders., Gesellschaft, Kultur, Theorie. Gesammelte Aufsätze zur neueren Geschichte (Göttingen 1976) 174-205. Ferner v. Otto Dann (Hrsg.), Vereinswesen und bürgerliche Gesellschaft in Deutschland (München 1984) (darin insbes. Wolfgang Hardtwig, Strukturmerkmale und Entwicklungstendenzen des Vereinswesens in Deutschland 1789-1848, 11-50) u. Etienne François (Hrsg.), Geselligkeit, Vereinswesen und bürgerliche Gesellschaft in Frankreich, Deutschland und der Schweiz, 1750-1850 (Paris 1986) sowie als Fallstudien bes. Ingo Tornow, Das Münchner Vereinswesen in der ersten Hälfte des 19. Jahrhunderts (München 1977) u. Eberbard Illner, Bürgerliche Organisierung in Elberfeld 1775-1850 (Neustadt 1982); Hans Schwarz, Das Vereinswesen an der Saar bis zur Mitte des 19. Jahrhunderts - der Verein als Medium der sozialen Kommunikation (Saarbrücken 1992); s. a. u. Anm. 13.

${ }^{4}$ So noch sehr deutlich bei Percy Ernst Scbramm, der selber einer der ersten Hamburger Familien entstammte. Alle Hamburger, „vom Bürgermeister bis zum letzten Mann im Hafen“, seien „eines Standes“ gewesen: Hamburg, Deutschland und die Welt (München 1943) 27. Als Beleg für das Selbstverständnis der Hamburgischen Führungsschicht zitiert er an anderer Stelle den berühmten Ausspruch Johann Karl Daniel Curios aus dem Jahre 1803: „Wir haben keinen Adel, keine Patrizier, keine Sklaven, ja selbst nicht einmal Untertanen. Alle wirklichen Hamburger kennen und haben nur einen einzigen Stand, den Stand des Bürgers": Neun Generationen. Dreihundert Jahre deutscher „Kulturgeschichte" im Lichte der Schicksale einer Hamburger Bürgerfamilie (1648-1948) Bd. 1 (Göttingen 1963) 295. Ebenso äußerte sich auch Jobann Isaak von Gerning in seiner Beschreibung Frankfurts: „Der Adel ist hier an Rechten und Abgaben Bürger“: Skizze von Frankfurt am Main (Frankfurt am Main 1800) 14. 
Beispiel des vor 1806, im Alten Reich, weitgehend patrizisch regierten Frankfurt deutlich $^{5}$, traten in den Dienst der umliegenden monarchisch regierten Staaten und zogen sich weitgehend aus der Stadt und ihren neuen Führungszirkeln zurück. Andere paßten sich den neuen Verhältnissen ganz $a^{6}$ und unterwarfen sich und ihre Familien den Rekrutierungsmechanismen der neuen, vor allem kaufmännischen bürgerlichen Elite, mit der sie schon in der Vergangenheit oft aufs engste zusammengewirkt hatten - das Kölner „Patriziat“ etwa verzichtete 1796 demonstrativ auf seine Adelstitel ${ }^{7}$. Insgesamt und speziell für die hier gewählten Beispiele kann man sagen, daß die Frage der Zuordnung des ehemaligen Patriziats zwar im Einzelfall Schwierigkeiten bereiten mag, für die Analyse der Konstellation und der Entwicklung als ganze jedoch keine größeren Probleme aufwirft.

Die Beispiele, die im folgenden näher betrachtet werden sollen, nämlich München, Augsburg, Karlsruhe, Mannheim, Wiesbaden, Münster und ergänzend, mit Blick speziell auf das sogenannte Bildungsbürgertum, dann noch Göttingen, stammen allesamt aus einem Sample von 16 Städten, das im Rahmen eines Frankfurter Forschungsprojektes zum Thema „Stadt und Bürgertum im 19. Jahrhundert" in den letzten Jahren unter vielfältigen Aspekten untersucht worden ist ${ }^{8}$.

Das zentrale Kriterium für die Auswahl der genannten sechs Städte bildete die Tatsache, daß in ihnen der Anteil des Adels an den selbständigen Haushaltungen deutlich über dem Landesdurchschnitt des Adelsanteils ${ }^{9}$ lag und der Adel hier, da ja praktisch

5 Vgl. dazu Ralf Roth, ,...der blühende Handel macht uns alle glücklich...“. Frankfurt am Main in der Umbruchszeit 1780-1825, in: Lothar Gall (Hrsg.), Vom alten zum neuen Bürgertum. Die mitteleuropäische Stadt im Umbruch 1780-1820 (München 1991) 357-408.

${ }^{6}$ Anschauliche Beispiele bieten die Augsburger Patrizierfamilien Stetten und Langenmantel: Während die Stettens als Erben des Bankhauses Halder mit an die Spitze der neuen bürgerlichen Führungsschicht der Stadt traten, gingen fast alle männlichen Mitglieder der - verarmten - Familie Langenmantel in den bayerischen Staatsdienst. Vgl. zum Hintergrund und zu den Zusammenhängen Frank Möller, Bürgertum als Schutzgemeinschaft. Augsburg 1794-1818, in: Gall (Hrsg.), Vom alten zum neuen Bürgertum (wie Anm. 5), 559-603; vgl. in diesem Rahmen auch Gerhard Hirschmann, Das Nürnberger Patriziat im Königreich Bayern 1806-1918. Eine sozialgeschichtliche Untersuchung (Nürnberg 1971).

7 Josef Hansen, Quellen zur Geschichte des Rheinlandes im Zeitalter der Französischen Revolution 1780-1801. Bd.3 (Bonn 1935) $792 \mathrm{f}$. Vgl. zu Köln Gisela Mettele, Kölner Bürgertum in der Umbruchszeit (1776-1815), in: Gall (Hrsg.), Vom alten zum neuen Bürgertum (wie Anm. 5), 229-275.

${ }^{8}$ Vgl. zu den Auswahlkriterien und zu den Zielsetzungen Gall (Hrsg.), Vom alten zum neuen Bürgertum (wie Anm. 5), Einleitung. - Den Bearbeitern der hier ausgewählten Städte (Ralf Zerback, Frank Möller, Dieter Hein, Thomas Weichel, Susanne Kill, Regina Jeske) danke ich wie auch allen anderen Mitarbeitern des Projekts, die das Material ,ihrer ${ }^{\text {“ }}$ Städte mit Blick auf die hier verfolgte Fragestellung gesichtet haben, für die Zusammenstellung der einzelnen Datenreihen. Es erwies sich dabei im übrigen, wie sinnvoll das Prinzip war, die auf seriellen Quellen und Massendaten basierenden Dateien nicht zu stark themengebunden, sondern nach systematischen und übergreifenden Prinzipien und Kriterien aufzubauen: Auch für eine Fragestellung wie die hier verfolgte, die nicht im Hauptstrom des Forschungsprojektes lag, ließ sich das Material verhältnismäßig rasch und unkompliziert erheben.

${ }^{9}$ Die auf meist sehr unsicheren statistischen Grundlagen beruhenden Annahmen und Schätzungen für die einzelnen Regionen und Länder schwanken zwischen $0,3 \%$ und rund $1 \%$; vgl. zusammenfassend zuletzt Hans-Ulrich Webler, Deutsche Gesellschaftsgeschichte (München 1987) 
generell zur Oberschicht der jeweiligen Stadt zu rechnen, somit einen erheblichen Prozentsatz der gesellschaftlich führenden Kräfte der betreffenden Stadt stellte. Es war hier also mit einer breiten „Kontaktzone“ zwischen ihm und den bürgerlichen Führungsschichten zu rechnen, gleichsam fokussiert und in ihrem Charakter, in ihrer Struktur und Entwicklung ablesbar an der Zusammensetzung und Geschichte des oder der zentralen Vereine der jeweiligen Stadt.

An der Spitze dieser sechs Städte mit überproportionalem Adelsanteil an den selbständigen Haushaltungen ${ }^{10}$ in der ersten Hälfte des 19. Jahrhunderts standen Karlsruhe mit (1828) 4,55\% und Münster mit (um 1800) 4,4\%, dann (1835) sogar 5,0\%, gefolgt von München mit zu Beginn des Jahrhunderts rund 3,5\% und Mannheim mit 18153,5 und $18293,7 \%$. Wiesbaden und Augsburg hatten in den dreißiger Jahren einen Adelsanteil von rund 3\%, der im Falle Augsburgs Mitte des Jahrhunderts auf rund 5\% stieg. Zum Vergleich: Die zehn anderen Städte des erwähnten Samples, angefangen bei alten Handels- und Gewerbestädten wie Bremen, Frankfurt am Main ${ }^{11}$, Köln und Leipzig, über frühindustrielle Gewerbestädte wie Aachen und Heilbronn bis hin $\mathrm{zu}$ Industriestädten wie Dortmund hatten einen Adelsanteil von unter oder um $0,3 \%$ (Leipzig 1813, Bremen 1830, Aachen um 1850) bis zu höchstens rund $1 \%{ }^{12}$.

Da die zentralen bürgerlichen Vereine überall ausgesprochene Elitenvereine waren $^{13}$, d.h. ihre Mitglieder sich aus einer gesellschaftlich und ökonomisch herausgeho-

\section{Fortsetzung Fußnote von Seite 32}

Bd. 1, $140 \mathrm{ff}$. u. Bd. 2, $145 \mathrm{ff}$. Dabei kommen die einzelnen Autoren für das gleiche Gebiet oft zu sehr unterschiedlichen Ergebnissen, etwa Webler (Bd. 2, 149) und Demel (s. o. Anm. 1) für Bayern. ${ }^{10}$ Gewisse Verzerrungen muß man insofern in Kauf nehmen, als etwa in den Augsburger Adreßkalendern nur die bürgerlichen Haushaltsvorstände aufgeführt werden, aber alle adeligen Militärs. Für den Gesamtbefund, auf den es hier ankommt, sind das jedoch vernachlässigbare Momente.

${ }^{11}$ Hier abzüglich der Angehörigen des Patriziats der reichsstädtischen Zeit, die im weiteren in den Dienst der umliegenden Landesherrn und Staaten traten.

12 In der bereits 1776 gegründeten Leipziger „Harmonie“ befanden sich denn auch unter den insgesamt 401 Personen, die bis 1825 dem Verein beitraten, eben fünf Angehörige des Adels, und davon waren noch zwei geadelte Kaufleute. Unter den Gründungsmitgliedern des „Clubs Aachener Casino“, der 1804 ins Leben trat, fand sich kein Mitglied des Adels, und bis 1830 traten auch nur fünf Adlige dem Verein bei, der in diesem Jahr 128 Mitglieder zählte. In dem 1837 gegründeten zweiten großen Verein der Stadt, der „Erholung“, befanden sich unter den 109 Gründungsmitgliedern und den 550 Personen, die bis 1850 dem Verein beitraten, eben acht Angehörige des Adels - man kann nachvolkziehen, wie isoliert sich der Referendar Otto von Bismarck, der seine adlige Herkunft so sehr betonte, in der Aachener Gesellschaft fühlen mußte.

${ }^{13} \mathrm{Vgl}$. dazu im einzelnen die Untersuchungen in Gall(Hrsg.), Vom alten zum neuen Bürgertum (wie Anm. 5) sowie aus der inzwischen recht umfangreich gewordenen Literatur zur Geschichte der Vereine vor allem noch Otto Dann (Hrsg.), Lesegesellschaften und bürgerliche Emanzipation. Ein europäischer Vergleich (München 1981); ders., Die Lesegesellschaften des 18. Jahrhunderts und der gesellschaftliche Aufbruch des deutschen Bürgertums, in: Ulrich Herrmann (Hrsg.), Die Bildung des Bürgers. Die Formierung der bürgerlichen Gesellschaft und die Gebildeten im 18. Jahrhundert (Weinheim/Basel 1982) 100-118; Wolfgang Hardtwig, Strukturmerkmale und Entwicklungstendenzen des Vereinswesens in Deutschland 1789-1848, in: Dann (Hrsg.), Vereinswesen und bürgerliche Gesellschaft (wie Anm. 3) 11-50; Franşois (Hrsg.), Geselligkeit, Vereinswesen und bürgerliche Gesellschaft (wie Anm. 3); Hans Schmitt, Das Vereinsleben der Stadt Weinheim. Volkskundliche Untersuchung zum kulturellen Leben einer Mittelstadt (Weinheim 
benen Gruppe von 10, höchstens 15\% der selbständigen Haushaltungen rekrutierten, ist unmittelbar verständlich, warum der Adel, sprich adlige höhere Beamte, Offiziere und, wo vorhanden, Mitglieder der Hofgesellschaft, in allen angeführten sechs Städten einen hohen Anteil der Mitgliedschaft stellte. Dieser Anteil schwankte zwischen einem Maximum von mehr als 50\% („Harmonie“ in München 1812:54\%, „Auf der Insel“ in Augsburg 1807: 55\%, „Museum“ in München 1826:61\%) und einem Minimum von einem guten Viertel, wobei der Durchschnitt bei etwa einem Drittel lag. Mit anderen Worten: Der Adel als solcher bildete in allen diesen „bürgerlichen" Vereinen eine starke Fraktion, deren Einfluß und Bedeutung noch dadurch gesteigert wurde, daß er vielfach an der Vereinsspitze noch massiver vertreten war, so im Ausschuß der Münchener „Harmonie“ des Jahres 1812 mit 73\%, unter den Vorstehern des Münchener „Museums“ sogar mit 92\% ${ }^{14}$. Viel bedeutungsvoller aber war, daß die stärkste Fraktion in den meisten dieser Vereine bürgerliche Beamte und Offiziere bildeten, die mit ihren adligen Kollegen und Kameraden vielfach über eine geradezu überwältigende Mehrheit verfügte. Im Münchener „Museum“ betrug diese Mehrheit 1802 bei einem dann rasch steigenden Adelsanteil von zunáchst $27 \%$ nicht weniger als $83 \%$, im Schwesterverein „Harmonie“ $181267 \%$ bei einem Adelsanteil von insgesamt $54 \%$. Das Karlsruher „Museum“ setzte sich 1815 aus $66 \%$ adligen und bürgerlichen Beamten und Offizieren zusammen, das Wiesbadener "Casino“ aus rund $75 \%{ }^{15}$. Der schon 1775 gegründete Münsteraner „Civilclub“ zählte $181058 \%$ der Mitglieder aus diesem Bereich und hielt sich, bei leicht steigender Tendenz, die ganze erste Jahrhunderthälfte auf dieser Höhe ${ }^{16}$. In allen diesen Vereinen blieb der eigentliche Kern des aufsteigenden städtischen Bürgertums, das Wirtschaftsbürgertum, eine kleine Minderheit, in München von zunächst 6 bzw. 13\%, in Karlsruhe, Wiesbaden und Münster von 10 bis $11 \%$, mit dann nach 1815 allmählich, aber durchaus nicht markant ansteigender Tendenz. Das sogenannte Bildungsbürgertum, also Anwälte, Ärzte, Apotheker, Lehrer, Pfarrer usw., war etwas stärker vertreten, blieb aber gegenüber der vorherrschenden Fraktion der Beamten und Offiziere ebenfalls eher marginal. Dabei legt die Analyse des Verhaltens einzelner Mitglieder den Schluß nahe, daß sich die Vertreter des Bildungsbürgertums gesellschaftlich wie politisch meist an den Normen, den

\footnotetext{
Fortsetzung Fußnote von Seite 33

1963); Herbert Freudenthal, Vereine in Hamburg. Ein Beitrag zur Geschichte und Volkskunde der Geselligkeit (Hamburg 1968); Wolfgang Meyer, Das Vereinswesen der Stadt Nürnberg im 19. Jahrhundert (Nürnberg 1970); Tornow, Das Münchner Vereinswesen (wie Anm. 3); Illner, Bürgerliche Organisierung (wie Anm. 3).

${ }^{14} \mathrm{Vgl}$. zu München in dieser Zeit Ralf Zerback, Zwischen Residenz und Rathaus. Bürgertum in München 1780-1820, in: Gall(Hrsg.), Vom alten zum neuen Bürgertum (wie Anm. 5), 605-653.

15 Vgl. zu Karlstuhe Dieter Hein, Umbruch und Aufbruch. Bürgertum in Karlsruhe und Mannheim 1780-1820, in: Gall (Hrsg.), Vom alten zum neuen Bürgertum (wie Anm. 5), 447-515; zu Wiesbaden Thomas Weichel, Die Kur- und Verwaltungsstadt Wiesbaden 1790-1822, in: ebd., 317-356.

${ }^{16} \mathrm{Zu}$ Münster Susanne Kill, Vom alten Münster zur preußischen Provinzialhauptstadt (17801816), in: Gall (Hrsg.), Vom alten zum neuen Bürgertum (wie Anm. 5), 105-141.
} 
Grundvorstellungen und auch dem Lebensstil der Beamtenschaft und des Offizierskorps orientierten, also diese Gruppe noch weiter verstärkten.

In den untersuchten Residenz- und Verwaltungsstädten, die oft zugleich Garnisonsstädte waren, waren also, so kann man zusammenfassend sagen, die zentralen „bürgerlichen" Vereine im wesentlichen Offiziers- und Beamtenvereine mit einem der Zeit entsprechend relativ hohen Adelsanteil und eher marginalen Berührungen mit dem eigentlichen städtischen Bürgertum, insbesondere dem Wirtschaftsbürgertum. Das hatte zur Folge, daß sich dieses Bürgertum im weiteren Verlauf vielfach in neuen bzw. im Ansatz stärker auf speziell „bürgerliche“ Bereiche konzentrierten Vereinen wie Lesegesellschaften, Bürger- und Schützenvereinen organisierte, die zugleich als Clearingstellen der Stadtpolitik im engeren Sinne dienen konnten, von der Beamte und Offiziere als „Eximierte“, als Einwohner ohne Bürgerrecht, ja mehrheitlich ausgeschlossen waren. Solche Vereine waren etwa der Münchener „Frohsinn“ von 1833, die Karlsruher Lesegesellschaft von 1816 oder der Wiesbadener „Altertumsverein“. Auch hier gab es zwar noch einen vergleichsweise hohen Adels- sowie Offiziers- und Beamtenanteil, beim „Frohsinn“ von $23 \%$ bzw. rund $50 \%$, im Wiesbadener „Altertumsverein“ von $19 \%$ bzw. $56 \%$. Aber nicht nur in den Relationen, sondern vor allem auch im Charakter und in den beteiligten Personen unterschieden sich diese Vereine doch von Anfang an sehr deutlich von den vorher genannten, so eindeutig vom Offizierskorps und der Beamtenschaft dominierten allgemeinen Vereinen. Dem entsprach dann auch die weitere Entwicklung, nämlich ein immer stärkeres Vordringen des Bildungs- und insbesondere des Wirtschaftsbürgertums. Im Wiesbadener „Altertumsverein “ beispielsweise sank der Anteil des Adels bis 1851 auf $8 \%$, der von Offizieren und Beamten insgesamt auf unter $40 \%$, während das Bildungs- und Wirtschaftsbürgertum nun klar die Mehrheit der Mitglieder stellte. Im „Casino“ hingegen behielten Offiziere und Beamte, bei sinkendem Adelsanteil - das entsprach der allgemeinen Entwicklung im Staatsdienst ${ }^{17}$ - auch nach der Jahrhundertmitte mit annähernd $70 \%$ ihre dominierende Stellung.

Was hier an Grundstrukturen und Entwicklungslinien des Verhältnisses von Adel und Stadtbürgertum an den Beispielen von München, Karlsruhe, Wiesbaden und Münster sichtbar wird, erschließt sich in seiner Bedeutung und in seiner Grundtendenz erst ganz, wenn man es mit den beiden anderen Städten mit deutlich erhöhtem Anteil des Adels an der Zahl der städtischen Haushaltungen, aber ganz anderer Struktur und Entwicklung vergleicht, nämlich mit Augsburg und Mannheim. In der alten Reichsstadt Augsburg ${ }^{18}$ mit ihrem Schwerpunkt auf Handwerk, Handel und Finanzwesen, die, wie erwähnt, 1830 einen Adelsanteil von 3\%, 1850 sogar von 5\% aufwies, gab es zwar auch in den drei zentralen Vereinen der Stadt „Auf der Insel“, „Harmonie“ und "Tivoli“ einen hohen Prozentsatz von Adligen, zunächst, zu Beginn des 19. Jahrhunderts, in dem Verein „Auf der Insel“ von rund $55 \%$, dann, in den dreißiger Jahren („Harmonie“ und „Tivoli“), von rund 40\%. Aber die Zusammensetzung dieses Adels war eine völlig andere als die in den genannten Residenz- und Verwaltungsstäd-

17 Vgl. speziell für Nassau, aber auch allgemein Eckhardt Treichel, Der Primat der Bürokratie. Bürokratischer Staat und bürokratische Elite im Herzogtum Nassau 1806-1866 (Stuttgart 1991).

${ }_{18}$ Vgl. dazu Möller, Bürgertum als Schutzgemeinschaft (wie Anm. 6). 
ten. Nur 19\% der großen Adelsfraktion des Vereins „Auf der Insel“ waren Offiziere oder Beamte, $37 \%$ gaben keinen Beruf an, waren also wohl in der Stadt wohnende Grundbesitzer, und 32\% zählten ökonomisch, d.h. als Bankiers und Kaufleute, zum Wirtschaftsbürgertum, verstärkten deren von Anfang an rund ein Drittel umfassende Fraktion zur Mehrheitsgruppe - mit den Vertretern des Bildungsbürgertums, von denen die Hälfte adlige Geistliche waren, zählten annähernd zwei Drittel der Mitglieder zum Stadtbürgertum im engeren Sinne. In der „Harmonie“ (Stand 1833) waren die Verhältnisse noch eindeutiger. Der Adelsanteil betrug hier nur noch rund 37\%, wovon wiederum rund $30 \%$ ökonomisch zum Wirtschaftsbürgertum zu rechnen waren, davon nicht weniger als $18 \%$ zur Kategorie der Bankiers. In den beiden anderen Kategorien hatten sich die Relationen insofern verschoben, als rund $21 \%$ wahrscheinlich in der Stadt wohnende adlige Grundbesitzer waren und $46 \%$ Offiziere und Beamte. Deren Gesamtanteil aber war mit rund 22\% (,Auf der Insel“ 1807 13\%) mit den Verhältnissen in den genannten Residenz- und Verwaltungsstädten ganz unvergleichbar zur Erinnerung: In München betrug er zu diesem Zeitpunkt 65\% (1802 83), in Wiesbaden $79 \%$, in Münster $58 \%$, in Karlsruhe $181566 \%$. Das adlige und geadelte Augsburger Wirtschaftsbürgertum nahm dabei eine recht bezeichnende Zwischenstellung ein zwischen der breiten Schicht des Stadtbürgertums und dem beamteten und alten Adel. Die bayerische Politik einer Verschmelzung der sozialen Aufsteiger aus dem Bürgertum mit dem Altadel ${ }^{19}$ wurde zwar durchaus kritisch registriert. "Auch würde es der Majestät wahrscheinlich nicht anders als lieb seyn, dem nach und nach an der Metallischen Schwindsucht absterbenden Adel durch einige Dutzend Süsskind, Wohnlich und Schaezler recrutirt und aufgefrischt zu sehen“, kommentierte Johann Lorenz von Schaezler seine Nobilitierung ${ }^{20}$. Aber die damit verbundene Anerkennung und die Gleichstellung mit dem Adel wurden doch gern angenommen. Von einem Rückzug aus Handel und Gewerbe konnte jedoch, auch wenn oft gleichzeitig mit der Nobilitierung in größerem Maße Grundbesitz - zur materiellen Absicherung wie auch als Statussymbol - erworben wurde, im allgemeinen nicht die Rede sein. Seit Ende der dreißiger Jahre gehörten im Gegenteil gerade diese adligen Wirtschaftsbürger zu den wesentlichen Geldgebern und Gründervätern der Augsburger Industrie.

Wie sehr das adlige Wirtschaftsbürgertum auch weiterhin dem übrigen städtischen Bürgertum verbunden blieb und auch gesellschaftlichen Kontakt zu den Spitzen der Handwerkerschaft hielt, zeigt eine Analyse der Zusammensetzung des „Schießgrabenvereins“. In diesem Verein mit 70\% städtischem Bürgertum aus Handel und Gewerbe zählen jene führenden Augsburger Bankiers-, Kaufmanns- und Fabrikantenfamilien ebenfalls zu den Mitgliedern. Im Lauf der nächsten Jahrzehnte gingen dann auch in Augsburg sowohl der Adels- als auch der Offiziers- und Beamtenanteil an den Mitgliedern der großen Vereine kontinuierlich zurück, und zwar zugunsten des immer eindeutiger dominierenden Wirtschaftsbürgertums. Mit anderen Worten, von einem dau-

19 Vgl. dazu Walter Demel, Die wirtschaftliche Lage des bayerischen Adels in den ersten Jahrzehnten des 19. Jahrhunderts, in: Armgard von Reden-Dobnal Ralph Melville(Hrsg.), Der Adel an der Schwelle des bürgerlichen Zeitalters 1780-1860 (Stuttgart 1988) 237-269.

${ }^{20}$ Zit. n. Woifgang Zorn, Handels- und Industriegeschichte Bayerisch-Schwabens 1648-1870 (Augsburg 1961) 254 . 
erhafteren Elitenkompromiß kann man auf der städtischen Ebene nicht sprechen, allerdings, so eng verzahnt wie Adel und die führende Gruppe des Wirtschaftsbürgertums hier von Anfang an waren, auch nicht von einem Elitenkonflikt, sondern von einer in vergleichsweise raschem Tempo verlaufenden Ablösung einer Elite durch eine andere, was die Mehrheitsverhältnisse und die Führungsposition angeht.

Etwas anders, aber in der Grundtendenz und in den Ergebnissen doch sehr ähnlich verlief die Entwicklung in der ehemaligen kurpfälzischen Residenzstadt Mannheim, die in wenigen Jahrzehnten zu einem Handelszentrum am Oberrhein und im weiteren dann auch zu einem Industriezentrum wurde ${ }^{21}$. Hier belief sich zu Beginn des 19. Jahrhunderts der Adelsanteil in den beiden großen Vereinen der Stadt, dem „Casino“ und dem 1808 davon abgespaltenen "Museum“ - 1814 vereinigten sich beide wieder in der „Harmonie“ - auf $31 \%$ bzw. 34\%, also rund ein Drittel, wobei die ganz große Mehrheit dieser adligen Mitglieder Offiziere und Beamte waren. Offiziere und Beamte stellten auch, wie in München und Karlsruhe, Wiesbaden und Münster, zunächst mit 55 bzw. $57 \%$ die Mehrheit sämtlicher Mitglieder, allerdings nicht in so ausgeprägter Form wie in den eben genannten Städten, was aus der Tatsache leicht erklärlich ist, daß Mannheim seit 1778 nicht mehr Residenz und seit dem Übergang der Kurpfalz an Baden 1803 auch nur noch begrenzt ein regionales Verwaltungszentrum bzw. eine Garnisonsstadt war. Andererseits ergab es sich aus der glanzvollen Vergangenheit der Residenzstadt mit ihrer intensiven Förderung aller Künste und Wissenschaften vor allem in der Regierungszeit Karl Theodors mit der Gründung der Akademie und schließlich des Nationaltheaters als weithin beachteten Höhepunkten, daß das Bildungsbürgertum mit $24 \%$ bzw. $29 \%$ die zweitstärkste Fraktion bildete - das Wirtschaftsbürgertum folgte mit $20 \%$ bzw. im „Museum“ gar nur $10 \%$, wobei von den 20\% im „Casino“ ein knappes Drittel als Verleger, Kunst- und Buchhändler und akademisch Gebildete fast schon eher dem Bildungsbürgertum zuzurechnen war. Diese Relationen nun verschoben sich binnen weniger Jahrzehnte in geradezu dramatischer Weise. Der Anteil des Adels, der schon 1815 auf $25 \%$ zurückgegangen war, sank bis 1848 auf $6 \%$, der der Offiziere und Hof- und Staatsbeamten insgesamt auf $21 \%$; von ihnen war nur noch ein knappes Sechstel adlig. Der Anteil des Bildungsbürgertums hielt sich in etwa: Er betrug $181524 \%, 184822 \%$. Hingegen schnellte die Zahl der Mitglieder aus dem Wirtschaftsbürgertum über $28 \% 1815$ auf $55 \% 1848$ empor; 1880 betrug er schließlich $72 \%{ }^{22}$. Aus Vereinen, die wie in den anderen ge-

21 Vgl. dazu Hein, Umbruch und Aufbruch (wie Anm. 15).

22 Mannheim näherte sich damit auch in dieser Beziehung, allerdings von der Basis eines signifikant höheren Adelsanteils, den Verhältnissen, die in alten Handelsstädten wie Köln und Frankfurt am Main, von dem vollständig bürgerlichen Leipzig (s. o. Anm. 12) ganz zu schweigen, schon seit Jahrzehnten herrschten. Während der Adel in Köln während der reichsstädtischen Zeit in den Freimaureriogen noch zahlreich vertreten war - unter den Gründern der Dreikönigsloge befanden sich $177520(=31,25 \%$ ) Angehörige des Adels - spielte er in den Kölner Vereinen des 19. Jahrhunderts mit dem "Casino" an der Spitze praktisch keine Rolle mehr; eine Ausnahme machte der vom preußischen König protegierte, 1841 gegründete „Central-Dombau-Verein“, in dem der Adel wie auch im Vorstand mit rund $20 \%$ vertreten war. In Frankfurt am Main war der Anteil des Adels an dem zentralen Verein, der 1802 gegründeten Casinogesellschaft, anfangs insofern noch höher, als Mitglieder des Stadtpatriziats - das im Unterschied zum Kölner (s.o.) an 
nannten Städten von Beamten und Offizieren mit einem - in Mannheim zu Anfang höheren - Anteil von Bildungsbürgern bestimmt wurden und in denen der Adel auch zahlenmäßig eine erhebliche Rolle spielte, waren Vereine geworden, in denen das Wirtschaftsbürgertum immer mehr den Ton angab;-in dem in den dreißiger Jahren gegründeten neuen „Casino" war dieser Teil des städtischen Bürgertums mit $87 \%$, davon $81 \%$ Kaufleute, fast unter sich.

Man mag einwenden, daß sich in diesen Zahlen vor allem ein quantitativer Vorgang spiegele, also die exproportionale Zunahme der Zahl der Wirtschaftsbürger mit höherem Einkommen und Vermögen und das - relative - Zurückbleiben der Zahl der Beamten und Offiziere wie auch, wenngleich in geringerem Maße, der sogenannten Bildungsbürger. Ganz abgesehen davon, daß quantitative Verschiebungen dieses AusmaBes über kurz oder lang auch qualitative Veränderungen, Veränderungen im Stil, in den Interessenschwerpunkten, die in einer Gemeinschaft vorherrschen, auch im Verhältnis der Gruppen zueinander mit sich bringen, ist unübersehbar, daß die einzelnen Fraktionen sich als solche empfanden und verstanden und entsprechend agierten. Das gilt insbesondere für das Verhältnis zum Adel, sprich zu den adligen Mitgliedern. Deren Anteil an der jeweils gewählten Vereinsführung, der, wie schon erwähnt, etwa in den beiden großen Münchener Vereinen, der „Harmonie“ und dem „Museum“, mit 75 bzw. 92\% überproportional hoch war, sank in Mannheim, wo er zunächst dem Adelsanteil an der Gesamtmitgliedschaft in etwa entsprach, wie dieser dramatisch ab was, wenn man sich das übliche Verfahren einer Wieder- bzw. auch wiederholten Neuwahl bewährter und angesehener Mitglieder vor Augen hält, sehr klar erkennen läßt, daß hier der Anteil des Adels von den Mitgliedern sehr bewußt zurückgeführt wurde; in der Zeit zwischen 1851 und 1870 waren von den insgesamt 25 Vorstandsmitgliedern nur noch zwei Angehörige des Adels.

Die Tendenz wird noch deutlicher, wenn man berücksichtigt, daß gleichzeitig der Anteil der Beamten im Vorstand - an der Gesamtmitgliedschaft ging dieser Anteil gleichfalls zurück - überproportional hoch blieb, ja, eher noch anstieg. Der Grund ist bei näherer Betrachtung sehr klar: Es waren, gipfelnd im Vorfeld und während der sogenannten liberalen Ära in Baden in den sechziger und siebziger Jahren, Söhne des

\section{Fortsetzung Fußnote von Seite 37}

seinem Adelstitel festgehalten hatte - sich zunächst noch an dem Vereinsleben beteiligten. In dem Maße, in dem diese Gruppe sich, wie erwähnt, aus der Stadt zurückzog, vielfach um in den Dienst der umliegenden Höfe zu treten, in dem Maße reduzierte sich auch der Anteil des Adels an der Mitgliedschaft der Casinogesellschaft und der anderen städtischen Vereine, bei denen im übrigen die Zahl der adligen Mitglieder von vornherein geringer war; auch in der Loge „Zur Einigkeit" ging schon in der zweiten Hälfte des 18. Jahrhunderts der Adelsanteil kontinuierlich zurück, von 47,5\% 1742/46 über 25\% 1789 bis zu 10\% im Jahre 1810: Karl Demeter, Die Frankfurter Loge Einigkeit 1742-1966. Ein Beitrag zur deutschen Geistes- und Sozialgeschichte (Frankfurt am Main 1967) 52, 65, 75f., 195-198. Auch wenn das Personal des Bundestages nach 1815/16 sozusagen eine zusätzliche Ressource bildete, blieb der Adelsanteil in allen diesen Vereinen durchgängig und zum Teil erheblich unter $10 \%$, mit Ausnahme des „Vereins für Geschichte und Kunst", zu dessen 15,5\% adligen Mitgliedern 1854 auch der preußische Bundestagsgesandte Otto von Bismarck zählte. 
Stadtbürgertums, die für solche Aufgaben abkömmlicher waren als ihre Brüder und Vettern, die den meist kaufmännischen Beruf des Vaters ergriffen hatten.

Von einer Symbiose, von einer wechselseitigen Durchdringung der alten, aristokratischen und der wirtschaftsbürgerlichen Fraktion der neuen, bürgerlichen Elite, die im Zeichen des wirtschaftlichen Wandels und dann der industriellen Revolution besonders dramatisch emporkam und die bürgerliche Führungsschicht in den Städten vielerorts zunehmend dominierte, kann also, wie auf der einen Seite das Beispiel der Residenz- und Verwaltungsstädte, auf der anderen Seite Städte wie Augsburg und vor allem Mannheim zeigen, praktisch nicht die Rede sein; eine Untersuchung des Heiratsverhaltens würde, wie erste Analysen erkennen lassen, zu ganz ähnlichen Ergebnissen gelangen. $\mathrm{Zu}$ einer derartigen Symbiose, vorsichtiger gesagt einer näheren Verbindung zwischen alten und neuen Eliten kam es im wesentlichen nur auf der staatlich-bürokratischen Ebene mit Kontakten zu Teilen des Bildungsbürgertums, die diesem Milieu in Deutschland traditionell nahestanden. Mit anderen Worten: Es handelte sich um einen nicht nur sektoral, sondern sicher auch, etwa was das Konnubium angeht, im Ausmaß begrenzten Ausgleich zwischen alten und neuen Eliten im Bereich der Beamtenschaft und des Offizierskorps ${ }^{23}$, wobei der Adel oft bis Ende des Jahrhunderts, ja, bis zum Ersten Weltkrieg vielfach die Führung behielt - auf der Ebene der Bürokratie und der Armee insgesamt wie in den entsprechend zusammengesetzten Vereinen. Außerhalb dieses Bereiches kam es hingegen zu einer Ablösung dessen, was man mit Blick auf Wien, den zeitgenössischen Sprachgebrauch aufnehmend, die erste, von Adel und Hof dominierte Gesellschaft genannt hat, durch die „zweite“, in der das Wirtschaftsbürgertum, angeführt von großen Kaufleuten, dann zunehmend auch von erfolgreichen Industriellen, den Ton angab ${ }^{24}$.

Das sogenannte Bildungsbürgertum, sprich Pfarrer, Lehrer, Professoren, die zahlenmäßig dann stark zunehmenden Angehörigen der freien Berufe, nahmen zwischen

${ }^{23}$ Zahlreicher scheinen die Heiraten zwischen den Söhnen und Töchtern adliger bzw. geadelter Wirtschaftsbürger und adligen Beamten in Augsburg gewesen zu sein - nicht zuletzt vor dem Hintergrund der entsprechenden Elitenpolitik des bayerischen Hofes und der bayerischen Regierung und der Sonderstellung der früh industrialisierten alten Handels- und Gewerbestadt in einem zunächst noch überwiegend agrarischen Land; vgl. dazu auch allgemein Hans Hesselmann, Das Wirtschaftsbürgertum in Bayern 1890-1914. Ein Beitrag zur Analyse der Wechselbeziehungen zwischen Wirtschaft und Politik am Beispiel des Wirtschaftsbürgertums im Bayern der Prinzregentenzeit (Stuttgart 1985).

${ }^{24}$ Wie sehr sich dieses Bürgertum als die neue Führungsschicht, als die neue gesellschaftliche Elite verstand, die die alte, den Adel, zunehmend ablöse, hat vor allem das rheinische Wirtschaftsbürgertum schon früh vorgeführt. Aristokratie in dem „Sinne genommen“, so David Hansemann 1830 als ihr Sprecher selbstbewußt, „daß die Vermögenderen und Angeseheneren des Staates den meisten Einfluß haben sollen, ist [...] ganz mein System": Preußens Lage und Politik am Ende des Jahres 1830. Denkschrift an den preußischen König. Aachen Dezember 1830, zit. n. Josef Hansen, Rheinische Briefe und Akten zur Geschichte der politischen Bewegung 18301850. Bd. 1 (Essen 1919) 11-81, hier 57. Als eine solche neue Aristokratie handelte man, mit den Worten Rudolf Bochs, „an Adels statt": Grenzenloses Wachstum? Das rheinische Wirtschaftbürgertum und seine Industrialisierungsdebatte 1814-1857 (Göttingen 1991) 40. Stärker die Nähe zum Adel betonend: Peter Wende, Die Adelsdebatte der Paulskirche, in: Adolf M. Birke/Lothar Kettenacker (Hrsg.), Bürgertum, Adel und Monarchie. Wandel der Lebensformen im Zeitalter des bürgerlichen Nationalismus (München/London/New York 1989) 37-51. 
diesen beiden Fraktionen eine Mittelstellung ein. Wo sich die Waage eindeutig zu der einen Seite neigte, also in den Residenz-, Garnisons- und Verwaltungsstädten zugunsten der adlig-bürgerlichen Beamten- und Offiziersfraktion, in den meisten übrigen Städten zugunsten des Wirtschaftsbürgertums, da finden sich die Vertreter des Bildungsbürgertums regelmäßig auf Seiten der Mehrheit, diese zusätzlich verstärkend. Das war nicht nur ein Reflex der menschlichen Natur und zugleich Ausdruck der Prägung der betreffenden Milieus durch die Anschauungen, den Lebensstil, die Mentalität der jeweiligen Mehrheit. Es war auch das natürliche Ergebnis der spezifischen beruflichen Interessen, die mehrheitlich in dem einen Stadttypus eine ganz andere Ausrichtung hatten als in dem anderen.

Aufschlußreicher für bestimmte Grundhaltungen des sogenannten Bildungsbürgertums ist das Verhalten der Mehrheit seiner Mitglieder eigentlich nur dort, wo die Optionen relativ offen waren, man sich also entscheiden konnte, mit welcher der genannten Fraktionen man enger zusammengehen wollte - was dann auch für das hier zur Debatte stehende Verhältnis zum Adel als vielfach noch tonangebender Gruppe in der einen Fraktion, der Fraktion der Offiziere und Beamten, von Bedeutung war. In dem Sample der in dem erwähnten Frankfurter Projekt untersuchten Städte gilt das eigentlich nur für Göttingen - prinzipiell auch für Heidelberg, wo allerdings die Quellenlage in dieser Beziehung sehr schlecht ist - sprich für die Universitätsstädte als, wenn man so will, „Brutstätten“ und natürliche Vororte des Bildungsbürgertums. Auf deren Vereine und ihre Entwicklung soll daher zum Abschluß noch ein Blick geworfen werden.

Der Adelsanteil an den selbständigen Haushaltungen Göttingens ${ }^{25}$ schwankte durch das ganze 19. Jahrhundert hindurch zwischen $1,3 \%$ und $1,5 \%$, lag also um einiges über dem geschätzten Landesdurchschnitt; ähnliches galt für Heidelberg, wo er zunächst, Ausgang des 18. Jahrhunderts, also noch in der kurpfälzischen Zeit, bei $1,9 \%$ lag und dann auf knapp 1\% zurückging, um dann wieder über $1,4 \%$ und $1,5 \%$ auf $1,6 \%$ im Jahre 1870 anzusteigen ${ }^{26}$. Der zentrale Verein in Göttingen, der „Civilclub“, bietet, wie zu erwarten, insofern ein wesentlich anderes Bild als hier, im Gegensatz zu den bisher betrachteten Residenz- und Verwaltungsstädten auf der einen, den Handels- und Gewerbestädten auf der anderen Seite, das sogenannte Bildungsbürgertum, sprich vor allem Professoren und daneben als zunächst sehr starke Fraktion die Rechtsanwälte, von Anfang an die Mehrheit hatte und auch den Vorstand über die Jahre hin eindeutig dominierte. 1825 stellte es $40 \%$ der Mitglieder, $183537 \%$ und 1885 im Nachfolger des „Civilclubs“, im sehr viel kleineren „Herrenclub“, $41 \%$. Ihm zur Seite trat ein kontinuierlich wachsender Anteil von Angehörigen des Wirtschaftsbürgertums der Stadt von zunächst 19\%, dann 23\% und schließlich, 1885, 33\%. Wie üblich stellten davon die Kaufleute den Löwenanteil von kontinuierlich rund $40 \%$. Ebenso fügt es sich in das bereits bekannte Bild, daß der Adelsanteil an der Mitglied-

25 Vgl. zu Göttingen in diesem Zusammenhang Regina Jeske, „Ein behagliches, vergnügtes Leben, wenig berührt von den Stürmen der Zeit“. Die Universitätsstadt Göttingen 1789-1818, in: Gall (Hrsg.), Vom alten zum neuen Bürgertum (wie Anm. 5), 65-104.

${ }^{26}$ Zu Heidelberg Marie-Lise Weber, Heidelberg in der Umbruchszeit zwischen 1789 und 1819, in: Gall (Hrsg.), Vom alten zum neuen Bürgertum (wie Anm. 5), 409-446. 
schaft, dessen traditioneller sozialer Stellung entsprechend, im Vergleich zu seinem Anteil an den selbständigen Haushaltungen überproportional hoch war und daß dabei Offiziere und Beamte ${ }^{27}$ mit zunächst 60,1835 sogar $80 \%$ deutlich dominierten. Schon etwas aus dem Rahmen fällt, daß dieser Anteil nicht, wie sonst gemeinhin üblich, langsam bzw., zum Beispiel in einer Stadt wie Mannheim, dramatisch fiel, sondern anstieg, von $6 \%$ auf $8 \%$ und schließlich $16 \%$. Dabei muß man freilich die Tatsache in Rechnung stellen, daß der „Herrenclub“, wie gesagt, sehr viel kleiner, sprich exklusiver war, hier also schon der Zugang eines oder zweier neuer Mitglieder zu deutlichen prozentualen Verschiebungen führte. Hinzu kam, daß die Zahl der adligen Professoren erheblich zunahm. Daß die Zunahme des Adelsanteils jedoch auch etwas mit der Grundhaltung, den politischen und sozialen Grundeinstellungen der Mehrheit der Mitglieder, also insbesondere der bildungsbürgerlichen Mehrheitsfraktion zu tun hat, läßt sich nicht nur aus einer Reihe von Einzelzeugnissen, sondern vor allem auch aus einem Vergleich mit der Zusammensetzung eines Vereins schließen, der 1848 wie in vielen anderen Städten zur Unterstützung der Ziele des sogenannten Centrums in der Frankfurter Nationalversammlung, also der liberalen Mitte, gegründet worden war. In diesem "Constitutionellen Verein“, der immerhin rund dreihundert Mitglieder zählte, also in etwa die Größenordnung des „Civilclubs“ umfaßte, waren die Anteile der einzelnen Gruppen in charakteristischer Weise verschoben. Die klare Mehrheitsfraktion bildete hier das Wirtschaftsbürgertum der Stadt mit 44\%, wobei sich die politischen Allianzen, die sich im Lauf des Vormärz herausgebildet hatten ${ }^{28}$, sehr deutlich darin spiegelten, daß rund ein Drittel der Mitglieder dieser Gruppe Kaufleute, Fabrikanten oder Verleger waren und 55\% Handwerker und Kleinhändler. Das Bildungsbürgertum war hingegen, zieht man die im „Constitutionellen Verein“ mit $6 \%$ recht zahlreichen Studenten ab, die es in den bürgerlichen Vereinen nicht gab, nur mit $18 \%$ vertreten, der Adel mit knapp 3\%. Anders gewendet: Der „Civilclub“ und dann der „Herrenclub“ standen politisch und auch in ihrer gesellschaftlichen Grundhaltung offenbar deutlich rechts von der Mitte, versammelten eine eher konservative Elite. Ihre bildungsbürgerlichen Mitglieder neigten den Kräften der bestehenden Ordnung, zu denen der Adel wie Offiziere und Beamte auch im vormärzlichen Hannover mehrheitlich zu rechnen waren, weit mehr zu als denjenigen, die, gestützt auf die dynamischen Elemente innerhalb des städtischen Bürgertums, eine neue politische und gesellschaftliche Ordnung erstrebten: Aus dem „Civilclub“, in dem Bildungsbürger und Beamte über eine Mehrheit von annähernd drei Viertel verfügten, ist keine Äußerung gegen die Suspendierung der hannoverschen Verfassung im Jahre 1837 bekannt geworden, gegen die eine kleine, dann freilich sehr rasch in ganz Deutschland zu Ruhm gelangte Gruppe von sieben Professoren der Göttinger Universität mit den Brüdern Grimm und dem Historiker Georg Gottfried Gervinus an der Spitze so leidenschaftlich protestierte - damit zugleich die Legende nährend, daß auch die Professoren, sozusagen als Speerspitze und Vorbild des Bildungsbürgertums, mehrheitlich politisch

${ }^{27}$ Göttingen hatte sowohl eine Garnison als auch eine - im Jahre 1817 errichtete - Königliche Justizkanzlei.

${ }_{28}$ Vgl. dazu generell Lothar Gall, Bürgertum in Deutschland (Berlin 1989), hier bes. $228 \mathrm{ff}$. 
links gestanden hätten, prinzipiell reformorientiert gewesen seien ${ }^{29}$. Gerade auch der Göttinger „Civilclub“ zeigt u. E. das Gegenteil ${ }^{30}$.

Faßt man noch einmal zusammen, so zeigt auch das Göttinger Beispiel - in Heidelberg hat sich der dortige Adel offenbar am Vereinsleben so gut wie nicht beteiligt -, daß von einer wirklichen Symbiose, gar von einer Verschmelzung der traditionellen Elite, sprich des Adels, mit der mehrheitlich aus dem Schoß des Stadtbürgertums aufstrebenden neuen bürgerlichen .Elite kaum die Rede sein kann. Diese empfand sich vielmehr sehr deutlich als konkurrierende, eben als neue Elite und wurde darin von der anderen Seite, vom Adel, aber auch von der bürgerlichen Beamtenschaft und auch von den bürgerlichen Teilen des Offizierskorps dadurch noch zusätzlich bestärkt, daß diese sich speziell vom Wirtschaftsbürgertum in vielfältiger Weise, etwa was Heiraten, privaten gesellschaftlichen Verkehr oder erwünschte Berufsziele der Söhne angeht, abzugrenzen versuchten. Das entsprach auch allgemein der Haltung der Mehrheit des sogenannten Bildungsbürgertums überall dort, wo es nicht existentiell, das heißt von seinen Berufsinteressen wie von der Struktur und den Mehrheitsverhältnissen der jeweiligen Stadt, auf ein Zusammengehen und Zusammenwirken mit jenem Wirtschaftsbürgertum angewiesen war. Hier freilich sprachen sich seine Vertreter dann oft sehr grundsätzlich und nachdrücklich für einen offensiven Zusammenschluß aller bürgerlichen Fraktionen mit entschiedener Frontstellung gegen die Kräfte der Vergangenheit und der noch bestehenden Ordnung mit dem Adel an der Spitze aus, letzteres in deutlicher Anknüpfung an den bürgerlichen Antiaristokratismus des 18. Jahrhunderts. Zu ihnen zählten etwa Karl von Rotteck, Georg Gottfried Gervinus oder, in Preußen, Johann Jacoby; auch der alte Ernst Moritz Arndt hat in der Paulskirche so argumentiert. Als ihre Gegner betrachteten sie insbesondere auch jene, die innerhalb des bürgerlichen Lagers, speziell im und aus dem Kreis des Bildungsbürgertums - das dabei auch ganz gezielt die Berufsinteressen seiner Söhne vor Augen hatte - für einen Ausgleich und eine Symbiose zwischen den traditionellen und den neuen bürgerlichen Eliten eintraten und hierbei vor allem wieder an die bildungsbürgerlichen Eliten dachten. Damit befestige man, so ihr Gegenargument, nur die bestehenden Verhältnisse und mit ihnen die traditionelle Vorrangstellung des Adels, der den Bürger, auch den bürgerlichen Beamten und Offizier, in den entscheidenden Bereichen doch immer nur als Juniorpartner akzeptieren werde. Man verhindere so den Übergang zu einer zeitgemäßen politischen und gesellschaftlichen Ordnung. Nach 1848 und vor allem dann nach der Bismarckschen Wende in der deutschen Politik haben sich allerdings, nicht auf der Ebene der meisten Städte, aber auf der Ebene vieler Länder und vor allem des Gesamtstaates, des Reiches von 1871, für zwei Menschenalter jene durchgesetzt, die für einen Ausgleich und eine Symbiose der traditionellen und der neuen Führungsschichten eintraten und damit vor allem die Beamtenschaft, das Offi-

${ }^{29} \mathrm{Zu}$ einer Adresse der Universität an den König gegen die Aufhebung der Verfassung, wie sie von verschiedenen Seiten vorgeschlagen wurde, ist es bezeichnenderweise nicht gekommen. 30 Immerhin waren drei der Göttinger Sieben, nämlich Dahlmann, Ewald und Weber, Mitglieder des Klubs, und auch Zachariä, der schließlich als Abgeordneter in der Nationalversammlung saß. Im einzelnen wird man also sehr genau differenzieren müssen. Die Grundtendenz, auf die es hier ankommt, erscheint jedoch sehr klar und eindeutig. 
zierskorps und weite Kreise des Bildungsbürgertums in ihrer Grundhaltung bestimmten. Was sie predigten, erwies sich freilich immer deutlicher als Illusion - Max Weber hat es ihnen am Ende des Jahrhunderts und zu Beginn des neuen, des 20., ein ums andere $\mathrm{Mal}$ ins Stammbuch geschrieben. Aber das ist ein anderes Thema. 
